\title{
Evaluación y uso de un péptido sintético para la detección de anticuerpos específicos contra Plasmodium falciparum
}

\author{
Development and use of a synthetic peptide for the detection of specific \\ antibodies against Plasmodium falciparum
}

Sandra M. Barrera ${ }^{1}$, Adriana Cepeda ${ }^{1}$, Hernando Curtidor ${ }^{2,3}$, Yolanda Silva ${ }^{3}$

\section{Resumen}

Introducción. La malaria continúa siendo una de las enfermedades que causa mayor morbimortalidad a nivel mundial. Por esta razón es importante desarrollar herramientas diagnósticas eficaces que se implementen como estrategias para el control de la enfermedad. Objetivos. Estandarizar las condiciones del inmunoensayo enzimático (ELISA), para la detección de IgG específica contra Plasmodium falciparum en sueros de pacientes diagnosticados por gota gruesa con malaria no complicada por $P$. falciparum, empleando como antígeno un extracto proteico obtenido a partir de cultivo de $P$. falciparum o un péptido sintético derivado de la proteína de superficie de merozoito GLURP (del inglés: glutamate rich protein). Materiales y métodos. Para la estandarización de la técnica, se utilizaron 22 sueros de pacientes positivos para malaria por $P$. falciparum y 11 diagnosticados positivos para malaria por $P$. vivax utilizando la técnica de gota gruesa. Como controles negativos se utilizaron 44 sueros de individuos sanos. Los sueros fueron probados contra extracto de proteínas del parásito y el péptido sintético IMT 94 derivado de la proteína GLURP, para evaluar las concentraciones y las diluciones óptimas de cada componente del sistema. Para la validación de la técnica se utilizaron 251 sueros de pacientes positivos para $P$. falciparum y 44 sueros de individuos sanos, diagnosticados utilizando la técnica de gota gruesa. Resultados. La técnica estandarizada con el péptido sintético permitió observar diferencia significativa en el reconocimiento de sueros de pacientes, controles positivos y negativos por los antígenos (extracto de proteínas y péptidos sintéticos). Conclusiones. La metodología usada permite identificar la respuestas inmune específica contra $P$. falciparum.

Palabras claves: Malaria, péptidos sintéticos, IgG, Plasmodium falciparum, Inmunodiagnóstico, ELISA. 


\begin{abstract}
Introduction. Malaria continues being one of the diseases causing the greatest morbi-mortality around the world. For that reason, effective diagnostic tools must thus be developed which can be used in strategies for controlling the disease. Objectives. To standardise enzyme-linked immunosorbent assay (ELISA) conditions for detecting Plasmodium falciparum specific IgG in sera from patients diagnosed by thick smear as suffering non-complicated malaria caused by $P$. falciparum. A protein extract obtained from $P$. falciparum culture or a synthetic peptide derived from glutamate rich protein (GLURP) merozoite surface protein would be used as antigen. Materials and Methods. 22 serum samples from patients diagnosed as suffering from $P$. falciparum malaria, 11 serum samples from patients diagnosed as suffering from $P$. vivax and 44 from healthy donors, diagnosed by using the thick smear tecnique were used for standarising the technique. Serum samples were tested against parasite protein extract and GLURP-derived IMT 94 synthetic peptide for standardisign optimum dilutions and concentrations for each component in the system. 251 serum samples from patients diagnosed as suffering from P. falciparum malaria and 44 from healthy donors diagnosed by using the thick smear tecnique were used to validate the technique. Results. The technique led to significant differences being observed in antigens (protein extract and synthetic peptides) recognising serum from positive and negative patients and controls. Conclusions. The methodology used led to identifying specific immune response against P. falciparum.
\end{abstract}

Keywords: Malaria, synthetic peptides, IgG, Plasmodium falciparum, ELISA, Immunodiagnostic.

\section{Introducción}

La malaria es una enfermedad parasitaria que constituye un problema importante de salud pública en todo el mundo, no sólo por sus índices altos de morbilidad y las tasas de mortalidad, sino también por el aumento de factores de riesgo asociados con su transmisión, como son la pobreza, desnutrición, hacinamiento de las poblaciones, migraciones, cambio climático, abastecimiento de agua y resistencia a antimaláricos e insecticidas [1]. Según el reporte de la Organización Mundial de la Salud (OMS) de 2017, se estima que para el ańo 2016 el número de casos de malaria fue de 216 millones y ocurrieron cerca de 450.000 muertes. Actualmente la malaria está presente de forma endémica en 106 países [2].

La disponibilidad de métodos adecuados de diagnóstico es de gran importancia en la prevención, tratamiento y control de enfermedades parasita- rias. Los métodos usados tradicionalmente para el diagnóstico parasitológico han sido la gota gruesa (prueba de referencia o gold estándar), el inmunodiagnóstico y los inmunoenzimáticos y los basados en técnicas de biología molecular. Aunque se han logrado mejoras con el tiempo, el éxito de estas pruebas se ha basado en criterios como rentabilidad, sensibilidad, especificidad y sencillez. La aplicación de estas pruebas diagnósticas se hace teniendo en cuenta los requerimientos específicos de las diferentes poblaciones y objetivos de cada estudio realizado [3].

La medición de anticuerpos a través de técnicas de inmunodiagnóstico puede tener gran valor en el estudio sero-epidemiológico masivo para analizar retrospectivamente la evolución de la malaria, establecer tasas de endemicidad, determinar prevalencia y distribución por grupos de edad de las diferentes especies parasitarias y delimitar áreas de transmisión de malaria; así como la identificación 
y seguimiento de focos de transmisión persistente, identificación de especie parasitaria y tamización de donantes de sangre [4].

Los ensayos inmunoenzimáticos, se han convertido en las técnicas más utilizadas para la detección de anticuerpos o antígenos circulantes. Estas pruebas serológicas se basan en el uso de antígenos (crudos o purificados) derivados de los parásitos de interés. Sin embargo, el uso de preparaciones en crudo como fuente de antígenos y la presencia de poli-parasitismo en un mismo individuo puede llevar a inespecificidad de la prueba, debido principalmente a la presencia de reactividad cruzada con antígenos propios o de diferentes especies.

El antígeno es por lo tanto el componente clave de las diferentes pruebas de inmunodiagnóstico. De tal forma que la identificación, selección y utilización de antígenos purificados o producidos por ingeniería molecular o síntesis química ha sido un objetivo reciente en la búsqueda de más sensibilidad y especificidad en inmunoensayos y pruebas inmunodiagnósticas [5]. Las inmunoglobulinas (lg's) o anticuerpos, constituyen el componente humoral de la respuesta inmune específica en todos los vertebrados. En el humano se reconocen cinco clases o isotipos de lg's denominados $\lg G$, $\operatorname{Ig} \mathrm{A}, \lg \mathrm{M}, \lg \mathrm{D}$ e $\mathrm{IgE}$, diferenciables gracias a las propiedades estructurales y antigénicas particulares de sus cadenas pesadas [6].

La respuesta inmune contra malaria es compleja e insuficientemente comprendida, pero se sabe que la malaria induce aumento en la producción de IgE e IgG sugiriendo un papel importante en la patogénesis [6]. Para el diagnostico de malaria se ha usado principalmente $\mathrm{IgG}$ ya que presenta gran heterogeneidad estructural y funcional (probablemente la más notable de todas las proteínas séricas) y puede ser cuantificada mediante técnicas inmunoquímicas.

Con el ánimo de obtener una prueba específica, rápida y de bajo costo para el diagnóstico de malaria causada por $P$. falciparum, en este trabajo se utilizó un péptido sintético para confirmar la presencia de anticuerpos IgG específicos contra $P$. falciparum. El péptido tiene secuencia específica para $P$. falciparum y es derivado de la proteína de superficie del merozoito denominada glutamate rich protein (GLURP) descrita como marcador molecular de malaria y con propiedades antigénicas [7-9]. Encontramos que el uso del péptido permite identificar respuestas inmunes especie-específicas diferenciadas contra $P$. falciparum.

\section{Materiales y métodos}

\section{Recoleccion de las muestras}

Los sueros de 33 pacientes diagnosticados con malaria (22 por $P$. falciparum y 11 por $P$. vivax) suministrados por la Fundación Instituto de Inmunología de Colombia (FIDIC) fueron usados como controles positivos. Como controles negativos se utilizaron 44 sueros de funcionarios del Instituto Nacional de Salud (INS), que decidieron participar voluntariamente. Para validar el inmunoensayo con el péptido sintetico, se probaron 251 sueros de pacientes positivos para malaria por $P$. falciparum recolectados en el departamento de Córdoba (Colombia), entre los años 2009-2010 por el grupo de Bioquimica del Instituto Nacional de Salud (INS). El diagnóstico de todas las muestras se hizo por la técnica de gota gruesa.

\section{Obtención de extracto de proteinas del parasito (Ag)}

Para la obtención del extracto de proteínas del parásito en estadio de merozoito se realizó un cultivo asincrónico con mayor cantidad de formas maduras de Plasmodium falciparum, con la cepa Colombiana FCB2 (INS, CODIGO MNL-R03.000.3010-002) [10], utilizando sangre humana grupo $\mathrm{O} \mathrm{Rh}+$, suspendida al 5\%, en medio de cultivo RPMI-1640 suplementado con HEPES (25mM), NaHCO3 (32mM), glutatión reducido y suero humano inactivo, de acuerdo con el método de Trager y Jensen [11]. El crecimiento 
de los parásitos fue monitoreado por observación microscópica de extendidos teñidos con Giemsa. Cuando en el cultivo predominaron más del $20 \%$ de las formas maduras [12], se inició la preparación del antígeno. El sedimento de células parasitadas fue suspendido en $10 \mathrm{ml}$ de PBS (1X) estéril y centrifugado. Este procedimiento se repitió 5 veces y luego se adicionaron $15 \mathrm{ml}$ de saponina al $0,1 \%$, se centrifugó a $2800 \mathrm{~g}$ por $30 \mathrm{~min}$ a $4^{\circ} \mathrm{C}$; se descartó el sobrenadante y al nuevo sedimento se le realizaron 6 lavados con PBS estéril frío. El sedimento se resuspendió en $1 \mathrm{ml}$ de PBS que contenía un coctel de inhibidores de proteasas; la solución se sonicó por pulsos por 5 min y se dejó a $4^{\circ} \mathrm{C} / 4 \mathrm{~h}$; después de este tiempo el contenido se centrifugó a 6.000 rpm $(2800 \mathrm{~g})$ por $30 \mathrm{~min}$ a $4^{\circ} \mathrm{C}$. El sobrenadante fue esterilizado por filtración a través de membrana de $0,22 \mu \mathrm{m}$, se recolectó en tubos de $0,4 \mathrm{ml}$ y se almacenó a $70^{\circ} \mathrm{C}$ hasta su uso [13].

\section{Obtención de péptido sintético (IMT 94)}

El péptido IMT94 ( ${ }^{901}$ GDKNEKGQHEIVEVEEILPEG ${ }^{919}$ ) que corresponde a un fragmento de la proteína GLURP presente en $P$. falciparum $[14,15]$, fue sintetizado siguiendo el método de síntesis de péptidos en fase sólida (SPPS, por su sigla en inglés) $[16,17]$. Se siguió la estrategia t-Boc y resina metilbencidrilamina (BMHA, por sus siglas en inglés) (0.5 mequiv/g, Bachem). El péptido sintetizado se separó de la resina utilizando HF, como ha sido reportado [18] y caracterizado por RP-HPLC (LaChrom) y espectroscopia de masas MALDI-TOF (Bruker Daltonics).

\section{Ensayo de inmunoabsorción ligado a enzimas (ELISA) para detección de IgG especifica contra P. falciparum (extracto-péptido)}

La estandarizarización de la tecnica de ELISA [19] para la detección de IgG específica contra $P$. falciparum usando como antígenos extracto de proteínas del parásito (Ag) y el péptido sintético IMT94 (GLURP), se realizo en tres fases: se determinó la concentración óptima de antígeno (extracto/pépti- do), la dilución óptima de los sueros (muestras) y se llevó a cabo análisis de correlación y de la reproducibilidad de la técnica utilizando 251 sueros de pacientes diagnosticados como positivos y muestras negativas para malaria causada por P. falciparum.

\section{Determinación de las diluciones óptimas del suero}

Péptido sintético IMT94 (GLURP) y extracto de proteínas $(10 \mu \mathrm{g} / \mathrm{mL})$ [15] se incubaron en pozos de placas para ELISA (96 pozos) a $37^{\circ} \mathrm{C}$ por $1 \mathrm{~h}$, $18 \mathrm{~h}$ a $4^{\circ} \mathrm{C}$, y de nuevo a $37^{\circ} \mathrm{C}$ por $1 \mathrm{~h}$. Las placas se lavaron 5 veces con PBS-Tween $0,05 \%$ y se incubaron con $0,5 \%$ de leche descremada $(5 \%)$ en PBS-Tween $0,05 \%$ por $1 \mathrm{~h}$ a temperatura ambiente (TA). Los sueros (44 negativos, 22 para Pf y 11 para $P v$ ) fueron adicionados a la placa por duplicado en diferentes diluciones $(1: 100,1: 200,1: 300$ y $1: 400)$. Se incubó por $1 \mathrm{~h}$ a $37^{\circ} \mathrm{C}$ y se lavó 5 veces con PBS-Tween $0,05 \%$ para remover el exceso de anticuerpo no unido al antigeno. Se adicionó anticuerpo tipo IgG de cabra anti-IgG humano acoplado a peroxidasa (Vector Laboratories) diluido $1: 5000$, se incubó $1 \mathrm{~h}$ a $37^{\circ} \mathrm{C}$ y se realizaron 5 lavados. Se reveló con el kit Microwell Peroxidase Substrate System (TMB, KPL Laboratories) y se leyó la absorbancia a 620nm en un lector de ELISA (Lab Systems Multiskan MS). Como control de la técnica se utilizó leche descremada al 5\% en PBS y cada ensayo se realizó por triplicado.

\section{Determinación de la concentración optima de antígeno}

Se realizó una prueba de ELISA como la descrita en el paso anterior utilizando los diferentes sueros a la dilución que presenta mejor reconocimiento de los antígenos y antígeno (Extracto $\mathrm{Ag} /$ péptido IMT94) a tres diferentes concentraciones: $1 \mu \mathrm{g} /$ $\mathrm{ml}, 5 \mu \mathrm{g} / \mathrm{ml}$ y $10 \mu \mathrm{g} / \mathrm{ml}$. Como control de la técnica se utilizó leche descremada al 5\% en PBS y cada ensayo se realizó por triplicado. 


\section{Determinación del punto de corte}

El punto de corte para diferenciar el reconocimiento positivo del negativo se calculó como el promedio de las absorbancias de los 44 sueros negativos para $P$. falciparum \pm 2 desviaciones estándar.

\section{Estudio de correlación}

Se realizó una prueba de ELISA como la descrita arriba, utilizando 251 sueros positivos para $P$. falciparum a la dilución que presenta mejor reconocimiento de los antígenos y fijando el antígeno a la concentración determinada que presenta mejor reconocimiento por los sueros. En este ensayo se incluyeron 11 sueros negativos para $P$. falciparum y positivos para $P$. vivax (suministrados por FIDIC), así como 22 sueros adicionales positivos para $P$. falciparum y negativos para $P$. vivax. Para determinar las diferencias en el reconocimiento de los antíge- nos (Extracto Ag/péptido IMT94) por parte de los 251 sueros positivos para P. falciparum, respecto de los controles, se utilizó el test no-paramétrico de Kruskal Wallis. Un valor de $\mathrm{p}<0,05$ se consideró como significativo. Los análisis estadísticos se realizaron con el programa SPSS versión 20.

\section{Resultados}

\section{Dilución optima del suero}

La dilución optima del suero, se definió como aquella dilución de suero a la cual se observó una mayor diferencia entre los valores de densidad óptica (DO) de los sueros positivos y negativos, tanto para el extracto como para el péptido sintético. Se observa en la Figura 1 que con los sueros con dilución 1:200 se obtiene diferencias que permiten definir esta dilución como la óptima para la realización de la prueba.

Figura 1. Determinación de las diluciones óptimas de suero usando como antígeno extracto proteico. Dilución optima 1:200.
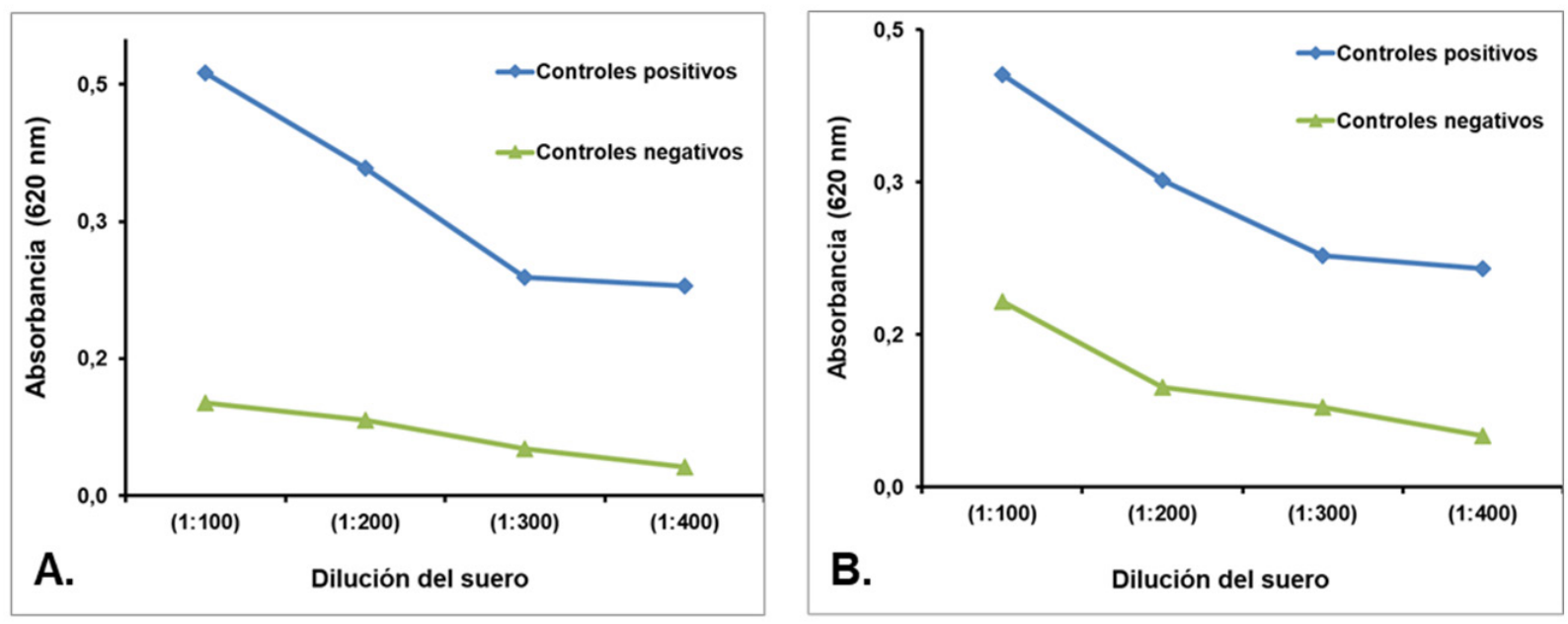

Fuente. Elaboración propia.

\section{Concentración optima de antígeno}

Se estableció la concentración mínima de antígeno a la cual se observa una mayor diferencia en los valores de DO, entre los sueros positivos y negativos, sin que variaciones importantes en la concentra- ción determinaran variaciones significativas en los valores de DO. La concentración óptima del antígeno tanto para el extracto del parásito como para el péptido sintético fue $10 \mu \mathrm{g} / \mathrm{ml}$, como se observa en la Figura 2. 
Figura 2. Determinación de la concentración optima de extracto. Concentración optima $10 \mu \mathrm{g} / \mathrm{ml}$
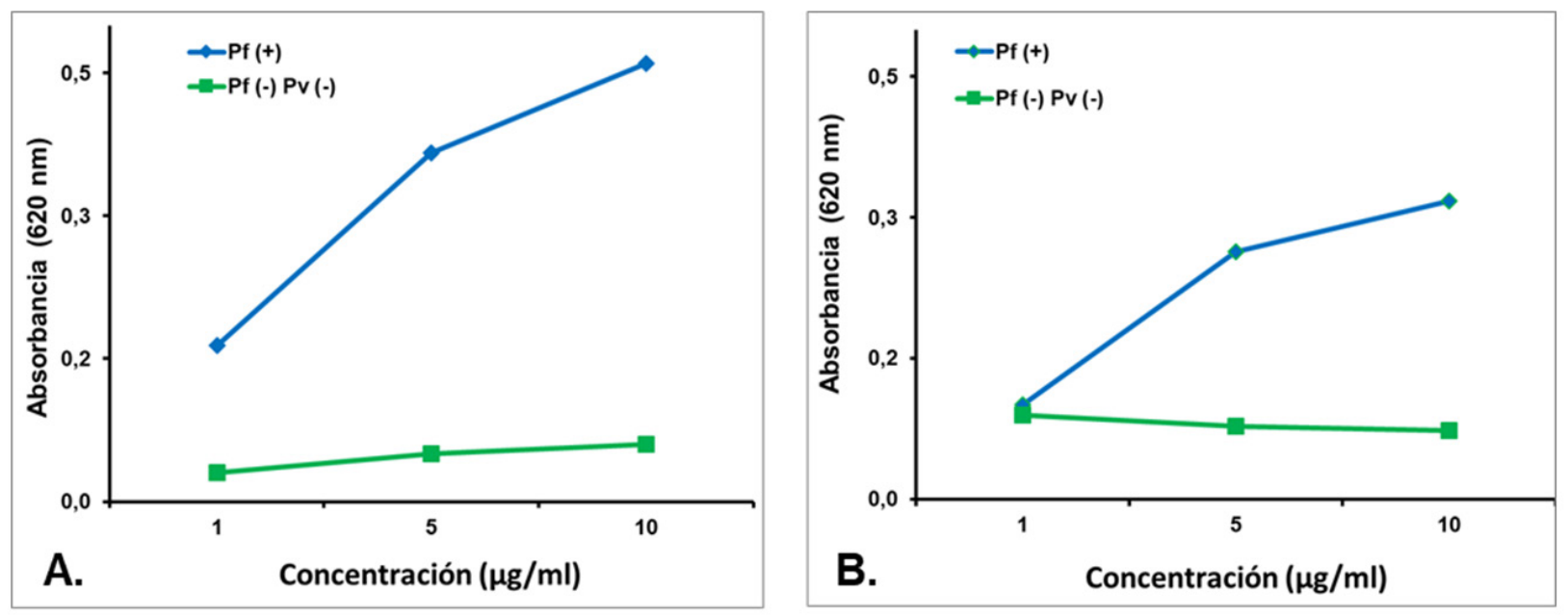

Fuente. Elaboración propia.

\section{Estandarización final}

Los sueros de 251 pacientes positivos para P. falci- y de 0,066-0,138 para el extracto y el péptido, resparum y los de 11 pacientes negativos para $P$. falci- pectivamente. Con los 251 sueros positivos para $P$. parum y positivos para $P$. vivax se probaron por la Falciparum, los rangos de DO fueron 0,231-0,751 técnica de ELISA en las condiciones óptimas de di- y 0,211-0,538 para el extracto y el péptido, respeclución (1:200) y concentración de antígeno en 10 tivamente. Los suero positivos para P. vivax pre$\mu \mathrm{g} / \mathrm{ml}$. Los rangos para los valores de DO obteni- sentaron valores bajo reconocimiento por los dos dos con los sueros negativos fueron de 0,108-0,193 antígenos (Figura 3).

Figura 3. Muestra el reconocimiento óptimo de las 251 muestras positivas para P. falciparum y controles negativos. A. Extracto del parasito. B. Péptido sintético.
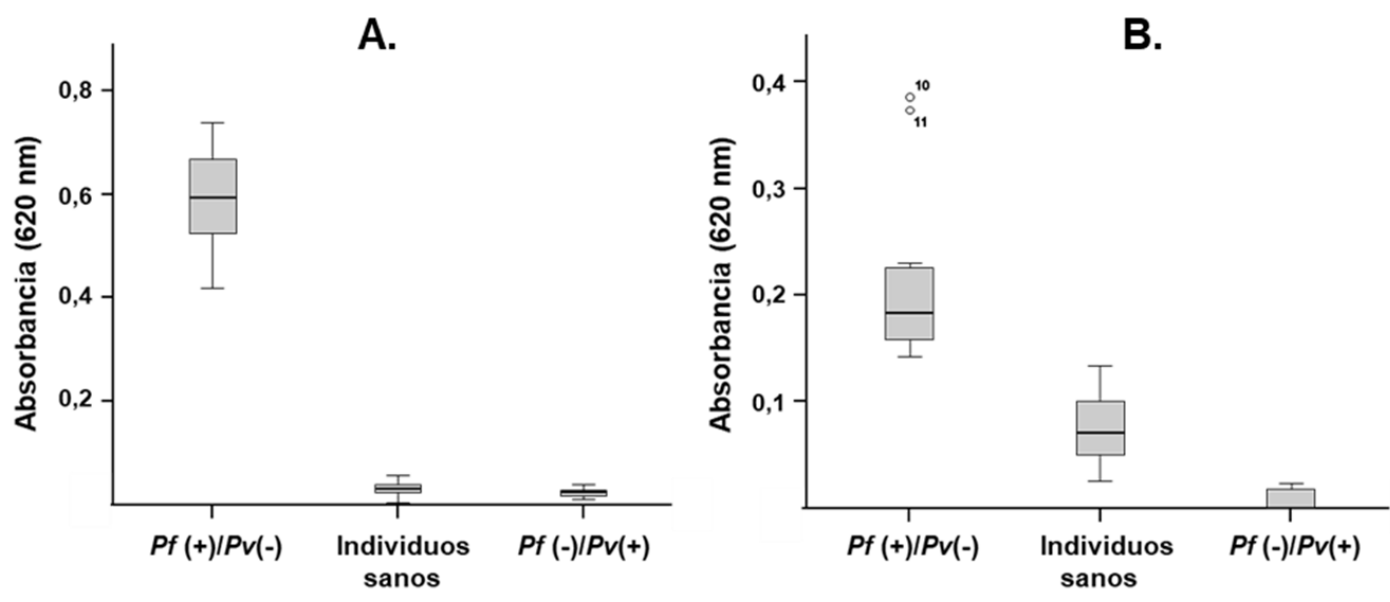

Fuente. Elaboración propia. 
Teniendo en cuenta el valor promedio de los valores de absorbancia de los 44 sueros utilizados como controles negativos, y el valor de su desviación estándar, el punto establecido de corte para el reconocimiento del extracto y del péptido se ubicó en 0,1 unidades de absorbancia.

\section{Análisis estadístico}

Para determinar las diferencias en el reconocimiento del extracto del parásito y del péptido entre los tres grupos evaluados (sueros positivos, sueros negativos y sueros positivos para P. vivax) se utilizó el test no-paramétrico de Kruskal Wallis. Fue considerado como estadísticamente significativo un valor de $\mathrm{p}<0,001$ tanto en el péptido como en el extracto. Estos análisis se realizaron con el programa SPSS versión 20.

\section{Discusión}

Hasta el momento no están disponibles comercialmente pruebas de diagnóstico para malaria basadas en péptidos sintéticos, pero hay resultados preliminares que confirman la posibilidad de desarrollo de pruebas basadas en la detección de anticuerpos y difusión de los antígenos. Este tipo de prueba sería de gran valor en el diagnóstico individual de la malaria en áreas no endémicas, así como en la delimitación de zonas de transmisión en los países endémicos. Por otro lado, el desarrollo de una prueba basada en la captura de antígeno sería de gran valor para diagnóstico rápido de las personas infectadas en áreas endémicas.

La malaria induce una respuesta inmune compleja e insuficientemente comprendida, con aumento en la producción de IgE e IgG. La estandarización del ensayo inmunoenzimático, se encaminó a confirmar la presencia de anticuerpos específicos contra Plasmodium falciparum en sueros de pacientes diagnosticados con malaria no complicada, empleando como antígeno un extracto proteico del parásito, estadio de trofozoito y un péptido sintético derivado de una proteína de superficie del merozoito denominada GLURP, permitiendo de esta manera realizar un diagnóstico especifico de malaria por P. falciparum. El objetivo fue estandarizar la técnica de ELISA, para determinar la respuesta inmune humoral mediante la detección de IgG específica contra $P$. falciparum. Para esto fue necesario seleccionar el antígeno adecuado para ser utilizado en la detección de IgG específica para P. falciparum (extracto proteico, péptido sintético), validar la técnica mediante ensayos por triplicado en muestras de pacientes positivos para malaria e individuos sanos y establecer los puntos de corte adecuados de detección de IgG.

El desarrollo de un método es un paso importante en el proceso de la mejora de todas las pruebas de diagnóstico basadas en síntesis de péptidos. Los resultados logrados en este trabajo muestran que tanto con el péptido sintético como con el extracto del parásito es posible obtener puntos de corte adecuados, ya que claramente permiten diferenciar los controles positivos de los negativos. El punto de corte se ubicó en 0,1 unidades de absorbancia, valor que permite definir como muestra positiva para IgG específica contra $P$. falciparum, las muestras que estén por encima de este valor. El punto de corte definido se obtuvo de acuerdo con 2 desviaciones estándar por arriba y por debajo de los valores obtenidos y que no se interpolaran entre muestras positivas y negativas. Además, al realizar el test no-paramétrico, se observaron diferencias estadísticamente significativas de $\mathrm{p}<0,001$ tanto usando como antígeno el péptido sintético como el extracto evidenciado claramente la diferencia entre muestras positivas como negativas (ver Figura 3).

Es de anotar que se presentó un mayor reconocimiento de los sueros cuando se utilizó el extracto como antígeno (rango DO 0,231 - 0,751), que cuando se usó el péptido sintético (rango DO 0,211 - 0,538). Esto se podría estar relacionado con la cantidad de antígenos en las muestras: mientras que en el extracto proteico se tienen un gran número de antígenos derivados de todas las proteínas presentes en el estadio de esquizonte, el 
péptido representa un solo antígeno. Estas diferencias en el reconocimiento de los antígenos evaluados por los sueros positivos y negativos para $P$. falciparum, muestran que el extracto proveniente directamente del cultivo del parásito presenta gran amplitud de respuesta comparada con el péptido, sin descartar que el péptido pueda emplearse como un buen marcador antigénico [20]. Interesantemente, el reconocimiento de los sueros $P f+$ por el péptido es mayor (rango de DO de 0,183-0,417) que el que se presenta por parte de los sueros de los voluntarios sanos y los sueros positivos para $P$. vivax (rango de DO 0,048 - 0,138) (ver Figura 4).

Figura 4. Reconocimiento del péptido sintetico por parte de los sueros usados como controles positivos $(P f(+) / P v(-))$, controles negativos sueros $P f(-) / P v(+)$ y $P f(-) / P v(-)$ (individuos sanos).

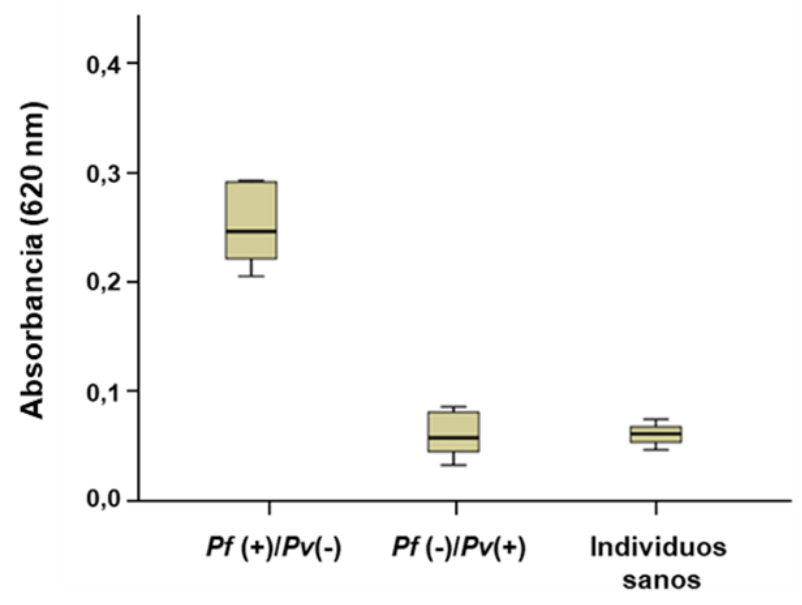

Fuente. Elaboración propia.

La metodología usada nos permite identificar respuestas inmunes especie-específicas, en particular, se puede diferenciar la respuesta inmune contra $P$. falciparum de la respuesta inmune contra $P$. vivax [21]. Los resultados permiten concluir que los dos antígenos utilizados (extracto proteico, péptido sintético) son útiles para la detección de IgG específica para $P$. falciparum, por las diferencias significativas evidentes entre las densidades ópticas obtenidas con los sueros de los pacientes diagnos- ticados por $P$. falciparum y los sueros de pacientes diagnosticados con P. vivax.

La prueba de ELISA estandarizada según el procedimiento aquí descrito, permitió obtener valores críticos de DO en muestras de suero, que permiten discriminar entre pacientes sanos y pacientes con malaria y se puede concluir que esta prueba bajo estas condiciones constituye una herramienta útil y confiable en el diagnóstico serológico para malaria. Es conveniente realizar ensayos en sueros de pacientes diagnosticados con otras especies de malaria para determinar si existe o no reacción cruzada.

\section{Conflicto de Intereses}

Los autores declaran que durante la realización del trabajo no existió conflicto de intereses.

\section{Referencias}

1. Lloveras, S.C., (Travellers to South America). Rev Chilena Infectol, 2011. 28(6): p. 520-8.

2. WHO, World malaria report 2017. World Health Organization, Geneva, Licence: CC BY-NC-SA 3.0 IGO., 2017.

3. Cerutti, C., Jr., et al., Epidemiologic aspects of the malaria transmission cycle in an area of very low incidence in Brazil. Malar J, 2007. 6: p. 33.

4. van Gool, T., et al., A simple and fast method to exclude high Plasmodium falciparum parasitaemia in travellers with imported malaria. Malar J, 2011. 10: p. 300.

5. Caballero, M.L., Inmunología de la infección por helmintos. Rev. Esp. Alergol Inmunol Clín., 1998. 13(6): p. 297-313.

6. Schroeder, H.W., Jr. and L. Cavacini, Structure and function of immunoglobulins. J Allergy Clin Immunol, 2010. 125(2 Suppl 2): p. S41-52.

7. Maestre, A., E. Arango, and J. Carmona-Fonseca, Status of allele frequency and diversity of Plasmodium falciparum msp1, msp 2 and glurp before implementation of an artemisinin-based combined therapy in Northwestern Colombia. Colomb Med (Cali), 2013. 44(4): p. 208-12.

8. Barrera, S.M., et al., (Genotypic survey of Plasmodium falciparum based on the msp1, msp2 and glurp genes by multiplex PCR). Biomedica, 2010. 30(4): p. 530-8. 
9. Amoah, L.E., et al., Natural antibody responses to Plasmodium falciparum MSP3 and GLURP(RO) antigens are associated with low parasite densities in malaria patients living in the Central Region of Ghana. Parasit Vectors, 2017. 10(1): p. 395.

10. Espinal Carlos, et al., Aislamiento y caracterización de cepas colombianas de Plasmodium falciparum. Biomédica, 1982. 2(3): p. 118-128.

11. Trager, W. and J.B. Jensen, Human malaria parasites in continuous culture. Science, 1976. 193(4254): p. 673-5.

12. Rojas, M.O. and M. Wasserman, Supersincronizacion del crecimiento in vitro del Plasmodium falciparum / Synchronization of in vitro growth of Plasmodium falciparum. Biomedica, 1987. 7(3/4): p. 75-80.

13. Heiber A and S. T., Preparation of Parasite Protein Extracts and Western Blot Analysis. Bio-protocol, 2014. 4(11).

14. Borre, M.B., et al., Primary structure and localization of a conserved immunogenic Plasmodium falciparum glutamate rich protein (GLURP) expressed in both the preerythrocytic and erythrocytic stages of the vertebrate life cycle. Mol Biochem Parasitol, 1991. 49(1): p. 119-31.

15. Noya, O., et al., Immunodiagnosis of parasitic diseases with synthetic peptides. Curr Protein Pept Sci, 2003. 4(4): p. 299-308.

16. Merrifield, R.B., Solid Phase Peptide Synthesis. I. The Synthesis of a Tetrapeptide. Journal of American Chemical Society, 1963. 85(14): p. 2149-2154.

17. Houghten, R.A., General method for the rapid solid-phase synthesis of large numbers of peptides: specificity of antigen-antibody interaction at the level of individual amino acids. Proc Natl Acad Sci U S A, 1985. 82(15): p. 5131-5.

18. Curtidor, H., et al., Plasmodium falciparum rhoptry neck protein 5 peptides bind to human red blood cells and inhibit parasite invasion. Peptides, 2014. 53: p. 210-7.

19. Pal-Bhowmick, I., et al., Generation and characterisation of monoclonal antibodies specific to Plasmodium falciparum enolase. J Vector Borne Dis, 2006. 43(2): p. 43-52.

20. Pinilla B Gladys, Chavarro P Bibiana, Moreno A Natalia, Navarrete O Jeannette, Muńoz M Liliana. Determinación de los genes, 16S ADNr, polA, y TpN47, en la detección de Treponema pallidum subsp. pallidum para el diagnóstico de sífilis congénita. Nova. 2015; $13(24$ ): 17-25.

21. Carrero Sandra Helena Suescún, HerediaMontoya Dina Paola, Bolańos Yoryany Mulato, Medellín Martín Orlando Pulido. Seroprevalencia de infección por Leptospira y factores de riesgo en estudiantes de una universidad de Colombia. Nova. 2017; 15( 27 ): 131-138. 